\title{
Architecting Systems for Value Robustness: Research Motivations and Progress
}

\author{
Adam M. Ross and Donna H. Rhodes \\ Massachusetts Institute of Technology \\ Building NE20-388, 77 Massachusetts Avenue \\ Cambridge, MA 02139 \\ http://seari.mit.edu
}

\begin{abstract}
The contemporary environment and large-scale systems challenges motivate research in support of a new paradigm: value robustness. Value robustness is defined as the ability of a system to continue to deliver stakeholder value in the face of changing contexts and needs. The authors' research seeks to develop methods for concept exploration, architecting, and design using a dynamic perspective for the purpose of realizing systems, products, and services that deliver sustained value to stakeholders in a changing world. The research is aimed at improving the development of real world systems and systems of systems, and involves deep engagement with government and industry stakeholders in the research conduct and the transition of research outcomes to industry practice.
\end{abstract}

Keywords - value robustness, system architecting, product architecting design for changeability, quantifying changeability, latent value

\section{INTRODUCTION}

Value robustness is the ability of a system to continue to deliver stakeholder value in the face of changing contexts and needs. The concept of value robustness is responsive to issues cited in a number of reports and studies on the challenges of systems acquisition over the past decade. In a workshop held several years ago, systems engineering experts explored the topic of engineering for a new type of "robustness," that is, developing systems capable of adapting to changes in missions and requirements; able to reliably function given changes in threats and environment; able to be easily modified to leverage new technologies; and scalable and adaptable $[1,28]$. The workshop prompted questions regarding what this implies for systems engineering, how this type of "robustness" can be measured, and who bears the cost.

A value-robust system is one that is perceived to be successful by stakeholders who continue to receive value from the system over time. It is important to note that value robustness can be achieved through system change or lack of system change [1]. For example, if stakeholder expectations increase, such as the need to have a longer system life, a value-robust system would be able to meet this new expectation, possibly achieved via over-design (requiring no change) or through life extension (requiring a system change).

Architecting value robust systems requires new methods for exploring the concept tradespace, as well as for decision making. Also needed are architecting principles and strategies, an approach for the quantification of changeability, and an improved ability for architects and analysts to classify value for purposes of dialogue and implementation. Ref. [1] provides foundational work in the architecting of systems for changeability as a means to realize value robustness. Selected projects within the authors' current research program are building upon this earlier work to evolve methods, strategies, and metrics for value robustness. The value robustness approach is being further validated and enhanced through multi-domain applications and case studies.

\section{RESEARCH LANDSCAPE}

The research landscape is the overall mental model under which research is formulated, performed, and transitioned to practice. An appropriate landscape is an important prerequisite for successful research endeavors. The academic environment within which research is undertaken has significant impact on formulation of research programs and their outcomes.

MIT's Engineering Systems Division (ESD) is a new kind of interdisciplinary academic unit that spans the departments within the School of Engineering, School of Science, School of Humanities, Arts, and Social Sciences, and Sloan School of Management. The academic unit provides a multi-disciplinary venue that is necessary for undertaking research in architecting systems using the value robustness paradigm. Engineering Systems is a field of study taking an integrative holistic view of large-scale, complex, technologically-enabled systems with significant enterprise level interactions and socio-technical interfaces, encompassing and also extending the footprint of systems engineering [2]. Research in engineering systems is interdisciplinary in nature, engaging faculty, researchers and students from a broad base of disciplines and domains. The engineering systems perspective is characterized by four unique perspectives that are important to value robustness research:

1. Broad interdisciplinary perspective that embraces technology, policy, management, and social science.

2. Intensified incorporation of system lifecycle properties, or "ilities," such as sustainability, safety and flexibility.

3. An emphasis on an enterprise perspective, acknowledging the interconnectedness of the "product system" with the enterprise system that develops and sustains it.

4. A complex synthesis of stakeholder perspectives, of which there may be conflicting and competing needs that must be resolved to serve the highest order system need.

The authors' research program, the Systems Engineering Advancement Research Initiative (SEAri), is part of the 
overall MIT ESD research agenda. SEAri's mission is to advance the theories, methods, and effective practice of systems engineering applied to complex socio-technical systems through collaborative research. One of the key goals for the research is to positively impact the thinking, approaches, and principles used by designers. Prescriptive methods seek to advance the state of the practice, and are based on sound principles and theories, but grounded by practical limitations and constraints. To develop prescriptive methods, sound normative principles and theories must be developed, in addition to descriptive knowledge regarding the current state of practice and constraints, as shown in Figure 1. The research involves deep engagement with industry and government projects, as described in Section V of the paper.

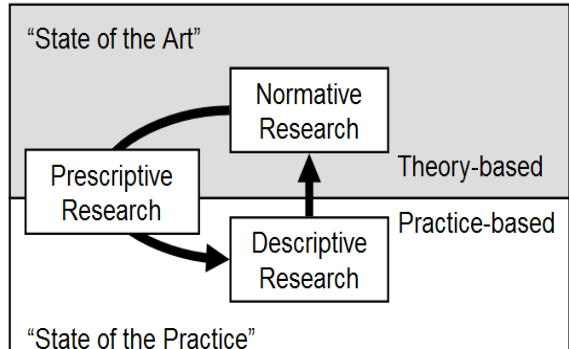

Fig. 1. Underlying Structure of SEAri Research Program.

\section{MOTIVATIONS}

A fundamental goal for systems engineering is to maximize the perception of system (or product) success by stakeholders. Success can be defined narrowly in terms of meeting performance, cost, and schedule expectations, or more broadly in terms of stakeholders perceiving benefit given the generalized costs of realizing the system. This broader goal of maximizing net benefit requires attention to how value is perceived by stakeholders through interaction with the system.

Understanding how people perceive value is fundamental to creating valuable systems [3]. This perspective is particularly important with regard to thinking about value delivery across the lifespan of a system. While an assumption of static needs, which is often captured in terms of requirements, simplifies the creation of systems and criteria for success, such an assumption is contrary to how people actually perceive value and will inhibit the realization of "valuable systems" as needs and perceptions shift with time.

Since needs are context dependent, affected by changing environments and limited access to information, the value perceptions of stakeholders will inevitably change with changes in context. Systems success will be difficult when the criteria for success - delivery of value--changes over time. One approach for dealing with this dynamic problem is to design systems for value robustness. When designers have a good grasp of the dynamic flow of value, they can develop truly long lasting high value systems. The role of a good designer is not about technical achievement, but about achieving value creation and sustainment using the proper terminology, methodology, and metrics. While foundational work has been accomplished, ongoing and planned research seeks to evolve and validate Design for Value Robustness through application across multiple domains.

\section{AREAS OF RESEARCH}

The value robustness research program seeks to develop methods for concept exploration, architecting, and design using a dynamic perspective for the purpose of realizing systems, products, and services that deliver sustained value to stakeholders in a changing world. This paper will highlight six areas within the overall research: (1) Methods for and applications of dynamic multi-attribute tradespace exploration; (2) Quantification of the changeability of system designs; (3) Architecting principles and strategies for survivable systems; (4) Dynamic tradespace exploration of systems of systems; (5) Techniques for the consideration of unarticulated and latent stakeholder value; and (6) Taxonomy for system 'ilities.'

\section{A. Dynamic Multi-attribute Tradespace Exploration Method}

The value-centric perspective is operationalized in conceptual design through the application of decision theoretic approaches (e.g., multi-attribute utility theory) to the engineering design process - making cost-benefit tradeoffs explicit in concept selection $[4,5]$. Traditional trade studies are insufficient for a comprehensive conceptual design effort as these consider a small number of alternative designs. Tradespace exploration builds on its application by adding computer-based parametric models and simulations, enabling comparison of hundreds or thousands of potential architectures, as shown in Figure $2[6,7]$.

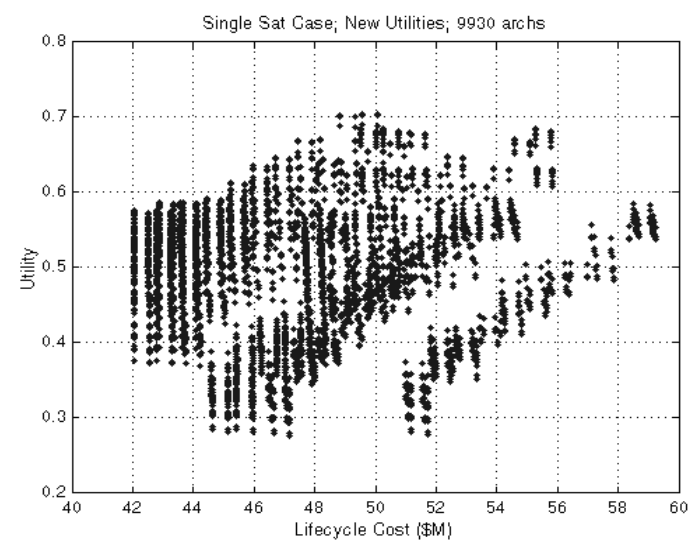

Fig 2. Tradespace Plot. Each point represents a design, and is plotted as utility versus cost

Tradespace exploration avoids the limits of local point solution trades by providing an understanding of the underlying relationship between the decision maker preference structure and potential designs [8]. Tradespace exploration may be used as a quantitative tool for evaluating the benefits, costs, and risks of alternative architectures - informing critical front-end decision making. In addition to evaluating potential technical capabilities, tradespaces may also be used to explore 
the implications of policy uncertainties [9] and changing value perceptions [1].

Tradespace exploration can be applied to the static case; however, higher benefit is achieved through dynamic tradespace exploration, wherein the tradespace is viewed as a network. Design transition rules can be applied to consider if and how to transition one design into another, enabling dynamic tradespace exploration as illustrated in Figure 3.

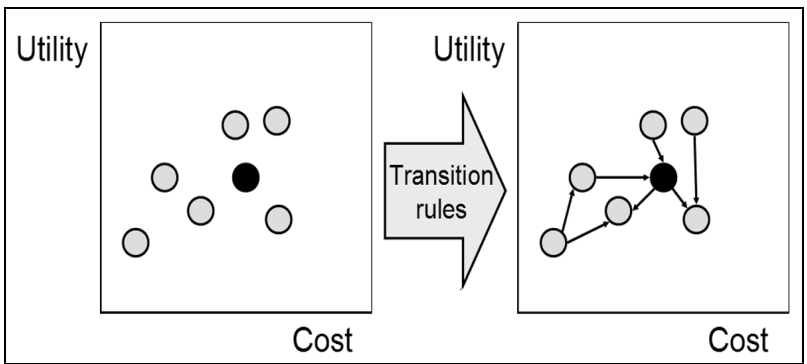

Fig. 3. Point designs in a tradespace can be linked as a network via transition rules to assess changeability.

Implications for Systems Engineering Practice. The application of tradespace exploration methodologies provides a means to more effectively understand tradeoffs between diverse stakeholder needs and possible design alternatives. The Multi-Attribute Tradespace Exploration (MATE) methodology was developed at MIT for exploring the tradespace of possible architectures rather than settling quickly on an optimum. The power of the method comes primarily from the ability to quantitatively assess many design choices very early in the design process. This ability allows designers and decision makers to explore many design options, and prevents focusing on a single "point design" too early. This capability enables quantitative assessment of factors such as variability in technical performance and cost, and impacts of changes in markets or policy, by allowing exploration of a large number of possible situations, including speculative ("what if") scenarios. Dynamic MATE [1] addresses designing for changeability to maintain delivered value in spite of system external changes. The method is suitable to application in multiple engineering domains, and has been demonstrated to improve design decision making. Cases have been undertaken primarily to 'single-system' military and space applications, and one current research project is looking at application of the method in the transportation domain. The overall implication of this research for practice is that designers will have an enhanced ability to consider concept alternatives in a rigorous way, not only for the present situation but also in considering futures where needs and contexts have shifted.

\section{B. Quantification of the Changeability of System Designs}

A related research topic is the quantification of changeability as a contribution to architecting science, involving methods that are rigorous and quantitative [10]. As described, the dynamic tradespace exploration method is predicated on linking designs in a tradespace network. If a design is considered to be a node in a design space of options, then the transition paths are arcs that connect that design to other design options. According to network theory, a representation of the possible future states for a transitioned design can be captured in terms of the outdegree of the design in a networked tradespace, which is a count of the number of outgoing arcs. However, counting the arcs alone is not enough to account for apparent disagreements between experts on the apparent changeability of a particular design. In order to reconcile the disagreement, only transitions at acceptable cost should be counted, thereby imposing a filter on the outdegree. A quantification of this subjective changeability is the Filtered Outdegree of a design within a networked tradespace. The Filtered Outdegree is formed through explicit consideration of transition paths between design instantiations limited by decision makers' subjective acceptability thresholds for "cost". The apparent changeability of a design will differ across decision makers based on their thresholds for acceptable transition cost.

Implications for Systems Engineering Practice. This research provides a construct for quantitatively assessing the changeability of candidate designs in tradespaces. A filter is applied based on the level of tolerance a decision maker has on "effort" to change (typically related to cost) to elucidate change paths that are viable. In Figure 4, the outdegree counts the total number of change paths from a given design (as shown by the number of arcs exiting the circle) where state 1 : $\mathrm{A}$, to future designs, states $2: \mathrm{A}^{\prime}, \mathrm{B}^{\prime}$, and $\mathrm{C}^{\prime}$ yields an outdegree of "four". The filtered outdegree counts the number of change paths with acceptable cost, from a given design. The illustration shows the acceptability threshold as indicated by the dotted box; therefore filtering out unacceptable change paths yields a filtered outdegree of "two."

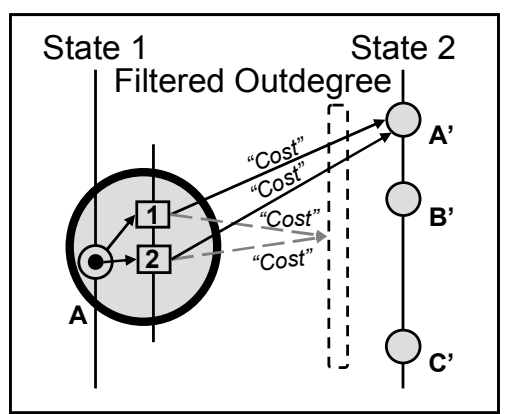

Fig. 4. Filtered Outdegree is a measure of changeability of a design as related to a decision maker's subjective threshold for acceptable "cost" of change, where cost may relate to dollars, time or other such factors

Use of this construct in tradespace exploration results in a metric for assessing the changeability of designs, giving designers an analytic construct for making design decisions, as well as the ability to compose repeatable and verifiable requirements for changeability in a design. 


\section{Architecting Principles and Strategies for Survivable Systems}

Survivability is the ability of a system to minimize the impact of a finite disturbance on value delivery. A third project in the area of value robustness seeks to develop and test a methodology for the conceptual design of survivable aerospace systems [11]. Survivability is an increasingly important attribute for systems that must be robust to environments characterized by system-threatening disturbance hazards. While disturbances may originate from a wide range of artificial and natural environments, a universal challenge confronting system architects is the specification, evaluation, and verification of systems with critical survivability requirements. Although survivability is an emergent system property that arises from interactions among components and between systems and their environments, conventional approaches to survivability engineering are often reductionist in nature (i.e., focused only on selected properties of subsystems or modules in isolation). Furthermore, existing survivability engineering methodologies are normally based on domain-specific operating scenarios and presupposed disturbances rather than a general theory with indeterminate threats. As a result, current methods neither accommodate dynamic threat environments nor facilitate communication among stakeholders trading system lifecycle cost, performance, and survivability.

In the first phase of the research, knowledge capture and synthesis, survivability is conceptualized as a value-centric, dynamic system property, generalizing existing definitions and theory. The second phase, theory development, explores distinguishing characteristics of survivability and the "ilities," enumerates survivability general design principles [12], and operationalizes survivability as a decision metric for tradespaces. The third phase, computer experimentation, tests the internal validity of the survivability metrics proposed during theory development. After empirically testing completeness of the survivability design principle set, the fourth phase, case applications, applies survivability methods and techniques to case studies. In particular, each case application includes formal interviews with system stakeholders to elicit multi-attribute utility functions; consultations with experts to gather sets of potential hostile operating environments; computer-based modeling and simulation to assess differential cost, performance, and survivability of candidate system architectures; and global sensitivity analysis across different system contexts.

Implications for Systems Engineering Practice. A first area of contribution to practice is a framework for precisely defining and relating survivability to other systems "ilities," along with a prescriptive set of general design principles, illustrated in Figure 5 in the context of temporal usefulness in a disturbance lifecycle. The implication for the practice is an improved ability to articulate, understand, and design survivable systems.

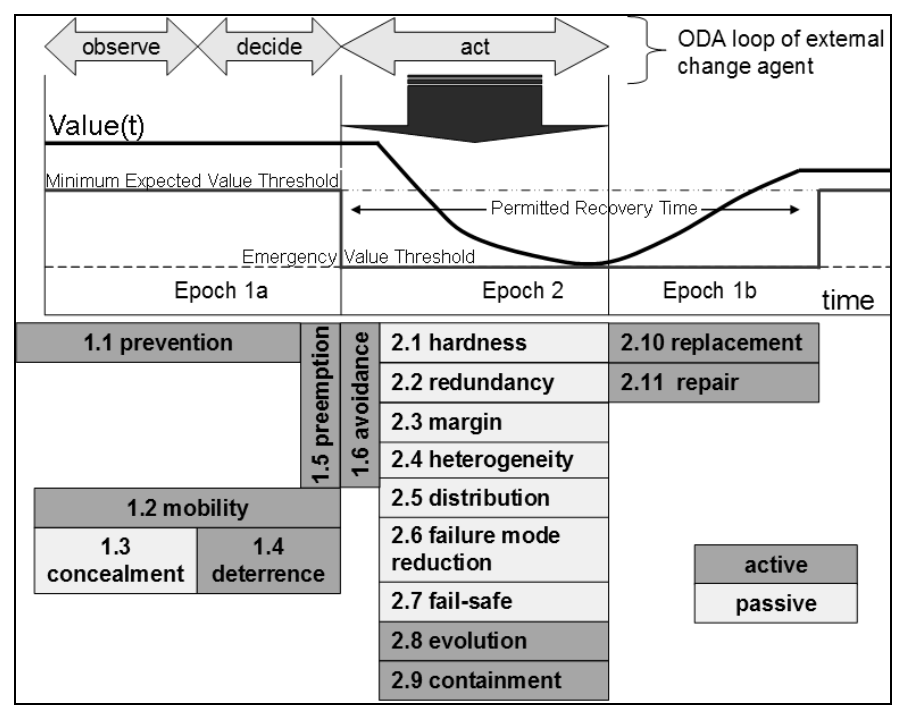

Fig. 5. Mapping of Preliminary Survivability Design Principle Set to Disturbance Lifecycle

A second area of contribution extends the dynamic multiattribute tradespace exploration to survivability. By making tradeoffs between cost, performance, and survivability explicit, the multi-dimensional tradespace provides a powerful framework for exploring a large set of alternative architectures. The expected result is an enhanced ability for system architects to communicate trades among cost, utility, and survivability to senior decision makers. This research focuses on aerospace and space systems, though it is expected that generalized principles will ultimately be derived from further research across domains.

\section{Dynamic Tradespace Exploration of Systems of Systems}

This fourth research area seeks to contribute to prescriptive design methods for Systems of Systems (SoS), building on existing tradespace exploration methods described in Section IV.A, extended for SoS considerations. Three key differences between SoS and traditional systems are considered in the research: stakeholder analysis, dynamics of SoS composition, and presence of legacy and new constituents.

Dynamic tradespace exploration is suitable for extension to SoS, as it encompasses desirable qualities. It allows for comparison of multiple concepts within the same tradespace, which is essential for SoS. As the method puts less emphasis on optimization, but rather provides a set of high benefit at cost solutions, the designer can observe the changes in benefits and costs that occur when the dynamic SoS changes. It provides a useful means to study changeability characteristics of the SoS over time, and can help identify SoS designs that are value robust to changes in constituent system membership, expectations, and contexts over time.

Due to a possibly large stakeholder set, a SoS designer is confronted with a complex multi-stakeholder problem during stakeholder analysis. Multi-stakeholder negotiations may require aggregating and trading the preferences of decision makers, depending on the relations between the constituent local and SoS global stakeholders. The designer must 
incorporate local and global distribution of costs and benefits into a multi-level value proposition for the SoS [13]. Studies to date have only considered a few stakeholders, usually focusing on the primary decision makers for the system $[8,14$, $15,16]$. In collaboration with an industry partner, a case study with a larger number of stakeholders is currently being investigated and will inform further development of the SoS tradespace method.

The dynamic tradespace exploration method accommodates changed expectation levels and design concepts very easily and quickly, enabling decisions for design or redesign of a SoS while it is in operation. Epoch-Era Analysis, shown in Figure 6, as part of the dynamic method [17], provides insight into when in the evolution of a SoS new systems may need to be added, and when investments should be made in new technologies. With Epoch-Era Analysis, the SoS lifetime is divided into a series of epochs, which are defined as time periods when significant system design characteristics, expectations, and context variables are fixed. Multiple consecutive epochs can be strung together to create an era, which represents a longer run view of the system evolution. Within each epoch, static analysis can be done to evaluate various designs. Significant changes in the SoS or the SoS context - such as a constituent system joining or leaving the SoS - can be represented by defining a new epoch. Path analysis within each epoch can help identify paths to SoS designs that provide high value delivery to the SoS stakeholders.

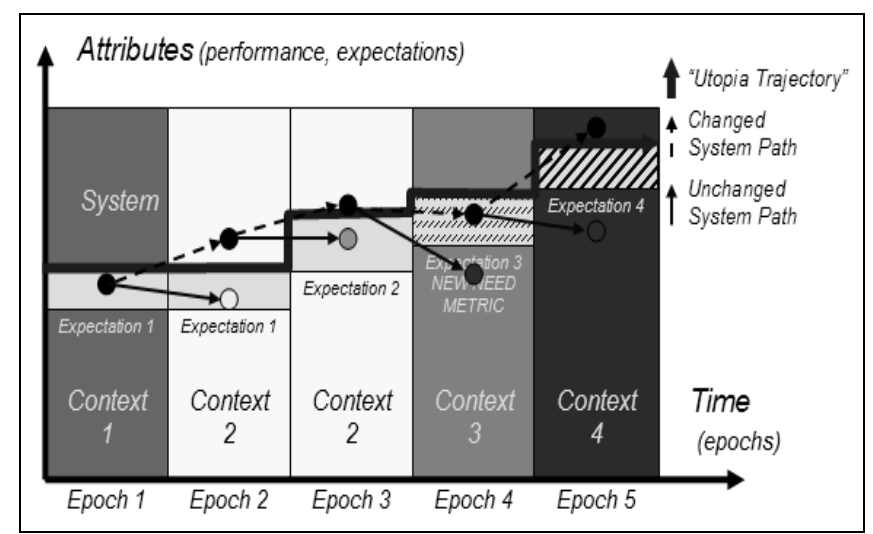

Fig 6. Epoch-Era Analysis. Each epoch has fixed context and expectations. Value of the system may degrade in a new epoch, but changing the system may restore value. Utopia trajectory is the optimal value delivery at least cost strategy across epochs.

Implications for Systems Engineering Practice. Over the last decade, the need for and interest in SoS has grown significantly. The US Department of Defense has recently increased its focus on methods of SoS design due to increased emphasis on integrating assets across forces and incorporating new technology to create multi-domain systems [18]. Multimodal transportation networks in the public sector are another example of SoS designs [19]. Many commercial product and service companies now invoke the SoS paradigm in moving toward integrated solution offerings.
SoS involve intensive decision making at multiple levels. Unlike traditional systems where tradespace exploration is most useful early in the lifecycle, SoS efforts require more continuous tradespace exploration as constituent systems enter and exit the SoS. SoS programs involve numerous and diverse decision makers, and creation of a shared value proposition necessitates a formal and rigorous approach to discussing and revealing SoS costs and benefits. Currently there is a lack of rigorous systems engineering methods for designing these types of complex, dynamic systems [20]. Many qualitative descriptions of SoS exist in the literature, but only heuristics and guiding principles have been suggested with regard to SoS design methods. New prescriptive approaches for addressing SoS problems through leveraging previous work in tradespace exploration will add significantly to the emerging practices for SoS engineering.

SoS are typically composed of both legacy and new systems, as well as existing and newly-designed interfaces between constituent systems. The SoS designer may not have the ability to affect enhancements and upgrades to legacy systems or interfaces. For the SoS designer, the 'system shell' concept [21] may be a useful construct when a constituent system design cannot be altered. By designing a shell, or wrapper, around the legacy system, it can more easily be integrated into the SoS and interfaced with other constituent systems without adversely affecting the legacy operation. This concept may also make it easier to switch components in and out of a SoS with minimum impact on the SoS operation.

Epoch-Era Analysis shows promise as a useful method for rapidly-evolving SoS, as the analysis can be quickly redone as strategic selection criteria and epoch boundary definitions change over time. This analysis may help identify SoS designs that are value robust to changes such as constituent systems joining and exiting the SoS, and help SoS designers devise strategies to transition to such designs.

\section{E. Unarticulated and Latent Value}

The design of value robust systems involves the elicitation of stakeholder values through direct means as well as through observing the system in use. In exploring design tradespaces, both unarticulated value, that which is not explicitly communicated to system designers, and dynamic value, that which changes over time, are not traditionally addressed through static analyses. Uncovering unarticulated values in the mind of stakeholders, as well as latent value in a system design will increase the likelihood that a system will continue to deliver value over time.

The discovery of latent value can have a positive impact on value robustness, and can additionally be a source of innovation. An example of this type of innovation is the experience of Nokia phone designers visiting in China, where it was observed that customers were using the light from their phone display in dark hallways in order to see and unlock doors. As a result, designers added a penlight to some phones as a new feature [22].

The research focuses on the approach of ensuring that system designers account for future changed value perceptions 
by thinking about these attributes according to the ease by which the system can display them. Since attributes can be on function or form, to "display" an attribute means that the system "does" or "exhibits" the attribute. For example, an attribute could be the color of the system, or the spatial resolution of the images it generates. The cost to display these attributes is how much it takes to either have or change color, or have or change an image spatial resolution. The attribute class spectrum from least to most costly include articulated "designed for" class 0 attributes, latent value class 1 attributes, combinatorial value class 2 attributes, accessible value class 3 attributes, and inaccessible value class 4 attributes [23].

Implications for Systems Engineering Practice. Over time, decision makers may change their mind on the attributes that provide value. The system that can change displayed attributes to match these new expectations will provide more value than a system with a fixed attribute set. Displaying matching attributes does not necessarily require a physical system change, especially if the system already contains latent value. More generally, however, a system may not have the attributes as latent value and must respond to changing expectations with system change.

The achievement of value robustness can be accomplished through either passive or active means. Passive value robustness can be achieved by developing "clever" systems, with a large set of latent value attributes, increasing the likelihood of being able to match new value expectations without requiring a system change. Active value robustness can be achieved through a strategy of pursuing designs with increased changeability and accessibility to likely high value regions of a tradespace. As value perceptions and expectations change, the active value robust system can change in order to display newly desired attributes.

The implications of this research are to improve the practice through more rigorous constructs that characterize system attributes in a spectrum in terms of their cost to display, including both articulated and potential attributes, such that designers can better make decisions. The ability to more effectively explore unarticulated and latent value can uncover essential needs and desires of stakeholders early in the process, which reduces dissatisfaction with the system later in time, as well as potentially increasing the latent value or decreasing the cost for a system to match newly revealed expectations. Observation of how stakeholders leverage latent value for systems in use or in early experimentation is an important source of innovation.

\section{F. Taxonomy for Enabling Stakeholder Dialogue on the Ilties}

While meeting requirements in a static context remains important, the performance of systems is increasingly defined by an ability to deliver value to stakeholders in the presence of changing operational environments, economic markets, and technological developments. Ref. [24] describes temporal system properties, the "ilities," as reflecting the degree to which systems are able to maintain or improve function in the presence of change, and emphasizes that the "ilities" constitute a rich research area for improving value delivery over the system lifecycle. The "ilities" are particularly critical to systems characterized by high cost, long lifecycles, high complexity, interdependencies with other systems, and dynamic operational contexts.

While most decision makers would agree that the "ilities" are important, they are neither well-defined nor easily evaluated in isolation. While some valuation methodologies do exist (e.g., real options for flexibility [25]), there is a need for a holistic framework for describing and evaluating systems with these properties. Each of these "ilities" has in common the concept of "change." It is the "what is changing" aspect that can be used to differentiate among the "ilities." Research has defined and elaborated a number of the "ilities," and is ongoing to conduct empirical descriptive studies of these in practice, with the intent to derive principles and insights into how these "ilities" interrelate [24]. In the research, each is examined in a rigorous and comprehensive manner, as described in Section IV.C for the case of survivability. Other examples of "ilities" of interest include:

1. Robustness is the ability of a system to maintain its level and set of specification parameters in the context of changing system external and internal forces. Robustness is determined by the sensitivity of chosen system specification parameters to context changes. A particular type of robustness, value robustness, is the ability of the system to maintain value delivery in the face of changing system external and internal forces, including stakeholder expectations.

2. Versatility is the ability of a system to satisfy diverse expectations on the system without the need for changing form. It is a measure of a system's inherent, or latent, value to a possibly diverse set of expectations over time.

3. Changeability is the ability of a system to alter its formand consequently possibly its function - at an acceptable level of resource expenditure (time, money, materials, and level of effort).

4. Flexibility is the ability of a system to be changed by a system-external change agent. Adaptability is the ability of a system to be changed by a system-internal change agent. The system boundary definition serves to distinguish between a flexible-type change and an adaptable-type change.

5. Scalability is the ability of a system to change the current level of a system specification parameter. Modifiability is the ability of a system to change the current set of system specification parameters.

Implications for Systems Engineering Practice. Through clarification of the variety of "ilities" in an analytic frame, a better dialogue is enabled among stakeholders, system architects, and analysts. The acquisition and development of systems is inhibited by lack of clarity in the definition and evaluation of "ilities". A Request for Proposal in the acquisition of a system may call for the system to be 
"flexible," but this property is ambiguous and not measurable. A first step to improving the engineering practice is to be able to have a precise dialogue about the desired system property, and to be able to specify it in unambiguous and quantitative terms. The taxonomy [10] can ultimately lead to the normative specification of the "ilities," such that prescriptive approaches can then be developed, including explicit specification, quantification, and verification of "ilities" system requirements.

\section{RESEARCH ENGAGEMENT MODEL}

The value robustness research involves many system stakeholders and necessitates an understanding of real world system contexts. Therefore, the authors have purposely architected the overall research program to involve deep engagement with sponsors and partners.

One ongoing project in partnership with a local defense company is focused on extending the dynamic tradespace exploration for SoS. While conducting research to enhance the methodology, there is a parallel effort focused on "tuning" the methodological approach for the industrial environment and its culture. In addition to enhancing the methodology itself, the project is resulting in learning about barriers and successful strategies for transitioning academic research to industry practice, and accommodating the cultural factors that come into play. By observing the real time interplay of practicing engineers and academic researchers, the result is new knowledge on the enablers for collaborative research, as well as a better method. The research serves a threefold objective: (1) to contribute to the sponsor's capabilities in tradespace exploration; (2) to further validate and enhance the MIT method; and (3) improve practices for collaborative research.

The SEAri research group embraces a philosophy of tightly coupling discovery and learning, with impact of research on art and practice. While researchers and students may engage in individual sponsored research projects, there is an emphasis on collaboration and knowledge sharing for synergistic research outcomes. Impact to practice is fostered through direct collaboration with corporate and government sponsors.

\section{DISCUSSION}

There are several new research directions that are planned for the overall value robustness area. Dynamic MATE has been applied to date in aerospace and space domains. A new project is applying the method to the transportation domain. In the future, researchers expect to apply this method to several other domains, which will serve to further develop and validate the method as generally useful and domain neutral [26]. Another planned research direction is to evolve characterization of architecture approaches for increasing changeability [27]. This work will ultimately lead to a designer's “toolkit” for changeable design.

Related research in the area of socio-technical decision making has resulted in an approach for achieving value robustness through the design of systems using natural value- centric time scales, as defined by their contexts, for conceptualizing system timelines. This approach, Epoch-Era Analysis, provides for visualization and a structured way to think about the temporal system value environment [17]. This type of analysis is central to the tradespace exploration process for system design comparison and selection, invoking passive or active value robustness design strategies. The analysis can also serve as a socio-technical bridge, integrating tradespace exploration activities of architects with those of analysts, which are often independent efforts. New research involves an in-depth application of Epoch-Era Analysis for enumerating many possible system futures in a case study for a US government agency, further evolving the underlying theory.

\section{CONCLUSIONS}

Value robustness has been defined as the ability of a system to continue to deliver stakeholder value in the face of changing contexts and needs. This concept is important for addressing challenges of the contemporary environment and large-scale systems [28]. The authors' research seeks to develop methods for concept exploration, architecting, and design using a dynamic perspective for the purpose of realizing systems, products, and services that deliver sustained value to stakeholders in a changing world. The research aims to improve the development of real world systems and systems of systems. It involves deep engagement with industry and government sponsors in the research conduct, as well as the transition of research outcomes to industry practice. As the world grows ever more complex at a faster rate, with new technologies and diverse stakeholder groups, interconnected systems, and the growth of more and more SoS, system designers will need to embrace designing for value robustness in order to ensure dynamic system success.

\section{ACKNOWLEDGMENT}

The authors gratefully acknowledge funding for this research provided through the MIT Systems Engineering Advancement Research Initiative (SEAri, http://seari.mit.edu), including sponsorship by Draper Laboratory, US Air Force Office of Scientific Research, MIT Portugal Program, National Science Foundation and a US Government Agency.

\section{REFERENCES}

[1] A.M. Ross, Managing Unarticulated Value: Changeability in MultiAttribute Tradespace Exploration, Doctor of Philosophy Dissertation, Engineering Systems Division, MIT, June 2006.

[2] D.H. Rhodes and D. Hastings, "The Case for Evolving Systems Engineering as a Field within Engineering Systems," MIT Engineering Systems Symposium, March 2004.

[3] A. Hall, A Methodology for Systems Engineering, NJ; Van Nostrand, 1962.

[4] R. Keeney and H. Raiffa, Decisions with Multiple Objectives Preference and Value Tradeoffs, Cambridge University Press, Cambridge UK, 1993.

[5] A. Sage and J. Armstrong, Introduction to Systems Engineering, Wiley \& Sons, New York, NY, 2000. 
[6] H. McManus, D. Hastings, and J. Warmkessel, "New Methods for Rapid Architecture Selection and Preliminary Design", Journal of Spacecraft and Rockets, Vol. 41, No 1, 2004, pp 10-19.

[7] A.M. Ross and D.E. Hastings, "The Tradespace Exploration Paradigm," INCOSE International Symposium 2005, Rochester, NY, July 2005.

[8] A.M. Ross, Multi-Attribute Tradespace Exploration with Concurrent Design as a Value-centric Framework for Space System Architecture and Design, Dual Master of Science Thesis, Aeronautics and Astronautics and Technology and Policy Program, MIT, June 2003.

[9] A. Weigel, Bringing Policy into Space Systems Conceptual Design: Quantitative and Qualitative Methods, PhD Dissertation, Technology, Management and Policy Program, MIT, June 2002.

[10] A.M. Ross, D.H. Rhodes, and D.E. Hastings, "Defining Changeability: Reconciling Flexibility, Adaptability, Scalability and Robustness for Maintaining Lifecycle Value," INCOSE International Symposium 2007, San Diego, CA, June 2007.

[11] M.G. Richards, D.E. Hastings, D.H. Rhodes, and A.L. Weigel, "Defining Survivability for Engineering Systems," 5th Conference on Systems Engineering Research, Hoboken, NJ, March 2007.

[12] M.G. Richards, A.M. Ross, D.E. Hastings, and D.H. Rhodes, "Design Principles for Survivable System Architecture," 1st Annual IEEE Systems Conference, Honolulu, HI, April 2007.

[13] N.B. Shah, D.E. Hastings, and D.H. Rhodes, "Systems of Systems and Emergent System Context," 5th Conference on Systems Engineering Research, Hoboken, NJ, March 2007.

[14] J.E. Derleth, Multi-Attribute Tradespace Exploration and Its Application to Evolutionary Acquisition, S.M., Massachusetts Institute of Technology, 2003.

[15] H. L. McManus and T.E. Schuman, "Understanding the Orbital Transfer Vehicle Trade Space", AIAA Space 2003 Conference and Exposition, Long Beach, CA, 2003.

[16] C.J. Roberts, Architecting Evolutionary Strategies Using Spiral Development for Space Based Radar, S.M. Thesis, Massachusetts Institute of Technology, 2003.

[17] A.M Ross and D.H. Rhodes, "Using Natural Value-Centric Time Scales for Conceptualizing System Timelines through Epoch-Era Analysis" SEAri Working Paper, WP-2007-1-3, 2007, http://seari.mit.edu, INCOSE International Symposium 2008, Utrecht, The Netherlands, June 2008 (forthcoming).

[18] DoD OuSD AT\&L, "System of Systems Engineering Guide: Considerations for Systems Engineering In a System of Systems Environment," Deputy Undersecretary of Defense (Acquisition and Technology), Office of the Undersecretary of Defense (Acquisition, Technology, and Logistics), v. 9, DoD, 2006.

[19] D. A. DeLaurentis, "Understanding Transportation as a System-ofSystems Design Problem", AIAA, Reno, Nevada, 2005.

[20] D. Chattopadhyay, A.M. Ross and D.H. Rhodes, "A Framework for Tradespace Exploration of Systems of Systems," 6th Conference on Systems Engineering Research, Los Angeles, CA, April 2008.

[21] A.M. Ross and D.H. Rhodes, "The System Shell as a Construct for Mitigating the Impact of Changing Contexts by Creating Opportunities for Value Robustness," 1st Annual IEEE Systems Conference, Honolulu, HI, April 2007.

[22] NY Times, "Hoping to Make Phone Buyers Flip", February 29, 2008

[23] A.M Ross and D.H. Rhodes, Using Attribute Classes to Uncover Latent Value during Conceptual Systems Design, $2^{\text {nd }}$ Annual IEEE Systems Conference, Montreal, Canada, April 4-5, 2008.

[24] H.M. McManus, M.G. Richards, A.M. Ross and D.E. Hastings, "A Framework for Incorporating "ilities" in Tradespace Studies," AIAA Space 2007, Long Beach, CA, September 2007.

[25] N. Shah, L. Viscito, J. Wilds, A.M. Ross and D. Hastings, "Quantifying Flexibility for Architecting Changeable Systems," 6th Conference on Systems Engineering Research, Los Angeles, CA, April 2008.

[26] J. Nickel, A.M. Ross, and D.H. Rhodes, Cross Domain Comparison of Design Factors in System Design and Analysis of Space and Transportation Systems, 6th Conference on Systems Engineering Research, April 2008.

[27] A.M. Ross and D.E. Hastings, "Assessing Changeability in Aerospace Systems Architecting and Design Using Dynamic Multi-Attribute Tradespace Exploration,” AIAA Space 2006, San Jose, CA, Sep 2006.
[28] D.H. Rhodes, Report on the Air Force/Lean Aerospace Initiative Workshop on Systems Engineering for Robustness, Massachusetts Institute of Technology, July 2004. 